\title{
Ischemic Acute Hepatic Failure - A Case Report and Review
}

\author{
Eduardo Cernadas ${ }^{*}$, Ana Catarina Dionisio, João Corrêa, Sara Ferreira, Dália Estevão, Leopoldina Vicente
}

Centro Hospitalar Universitário Cova da Beira, Covilha, Portugal

DOI: $10.36347 /$ sjmcr.2020.v08i03.044

| Received: 18.03.2020 | Accepted: 25.03.2020 | Published: 30.03.2020

*Corresponding author: Eduardo Cernadas

\section{Abstract}

Ischemic hepatitis is a relatively uncommon lesion representing 1 to $2.5 \%$ of total patients admitted to the Intensive Care Units (ICU). It is characterized by a massive but transient elevation of plasma aminotransferases caused by anoxic necrosis of centrolobular hepatocytes. It often develops in association with shock (septic but also hypovolemic, distributive or cardiogenic), heart failure and respiratory failure, and these etiologies account for about $90 \%$ of cases. Occasionally it may result in acute liver failure. The authors describe the case of a 70-year-old woman with a history of atrial fibrillation ( $\mathrm{AF}$ ) and heart failure (HF), referred to the Emergency Department (ED) for vomiting and abdominal pain. On admission the AF with rapid ventricular response motivated hospitalization in an Intermediate Care Unit. Progressive worsening with acute heart failure and hemodynamic instability, with consequent clear increase in aminotransferase values, were consistent with the diagnosis of ischemic hepatitis. In this context, the patient developed hepatic encephalopathy and elevation of the International Normalized Ratio (INR), whose etiology were attributed to the acute liver failure. After hemodynamic stabilization and heart failure compensation, the aim was to progressively normalize the state of consciousness of the patient as well as the liver enzymes and coagulation study.

Keywords: Internal Medicine; Intensive Care Medicine; Hepatic Encephalopathy; Ischemic Hepatitis; Acute Liver Failure; Acute Cardiac Failure.

Copyright @ 2020: This is an open-access article distributed under the terms of the Creative Commons Attribution license which permits unrestricted use, distribution, and reproduction in any medium for non-commercial use (NonCommercial, or CC-BY-NC) provided the original author and source are credited.

\section{INTRODUCTION}

The liver is an organ of high metabolic activity, with a blood flow ranging from 800 to $1200 \mathrm{ml}$ / min, which is about $20 \%$ to $25 \%$ of total cardiac output [1], making it particularly susceptible to hemodynamic changes.

There are several forms of hepatic vascular injury, ranging from hepatic vein thrombosis (BuddChiari syndrome; portal vein thrombosis; hepatic artery thrombosis; hepatocyte sinus obstruction syndrome; congestion due to heart failure or, more rarely, hepatic infarction and ischemic hepatitis[1].

Histologically, ischemic hepatitis is characterized by centrilobular necrosis of hepatocytes on zone three, without evidence of associated local inflammation. Regarding the clinical and laboratory findings that makes this diagnosis is the fulfilment of three major conditions: i) clinical heart, respiratory or circulatory failure that motivates the condition; ii) drastic increase in aminotransferases levels that can reach values up to 20 times higher than the upper normal limit iii) exclusion of other causes of acute liver necrosis, particularly viruses or drugs [1].
Although the pathophysiology is not yet clear, the proposed mechanisms of injury are mainly related to three etiologies: septic shock (50\%); heart and respiratory failure, which lead to hypoxemia and hepatocyte injury by anoxia, with consequent centrilobular necrosis, increased levels of aminotransferases according to a characteristic pattern in these cases.

The diffuse nature of the lesion throughout the parenchyma allows the differential diagnosis with hepatic infarction, which is characterized by a more localized lesion.

Acute liver failure is a rare but potentially fatal complication. It consists of rapid deterioration of liver function that results in coagulopathy (usually with a greater than 1.5) and a sudden change in consciousness of the patient. In the evaluation of patients with acute liver failure, it is crucial to identify the cause, as the delay in the institution of specific treatment may determine the prognosis [2, 3]. 
All patients with clinical and/or laboratory evidence of acute, moderate or severe hepatitis should have immediate and careful assessment of the state of consciousness and coagulation study (INR). The presence of deterioration of any of these parameters is a reason for hospital admission [3].

\section{Clinical Case}

The authors present the case of a 70-year-old female Caucasian patient, independent in her daily life activities, who recurred to the ED with a two day complaints of severe asthenia, nausea, vomiting and epigastralgia irradiating to the right hypochondrium. She had a known history of atrial fibrillation and heart failure with severe left ventricular (LV) disfunction ( $25 \% \mathrm{LV}$ ejection fraction). She was being treated on an outpatient basis with $0.125 \mathrm{mg} /$ day digoxin, $100 \mathrm{mg} /$ day metoprolol and $5 \mathrm{mg} /$ day ramipril. On admission to the ED she was conscious, collaborative, apparently without neurological signs, hydrated skin and mucosa, anicteric, acyanotic, eupneic at rest, tympanic temperature of $38.4^{\circ} \mathrm{C}$, tachycardic $(100-130 \mathrm{bpm})$, with arterial pressure of $101 / 82 \mathrm{mmHg}$. At abdominal palpation she complained of discomfort in the epigastric region and right hypochondrium, without defense: No palpable masses or organomegalies were detected. Electrocardiographic tracing showed AF with rapid ventricular response of $132 \mathrm{bpm}$. Analytically, acute renal injury (creatinine $1.76 \mathrm{mg} / \mathrm{dl}, \mathrm{U}$ 132), increased aminotransferases (AST $164 \mathrm{U} / \mathrm{L}$; ALT $139 \mathrm{U} / \mathrm{L}$ ), LDH 982 U / L and INR (5.1). Pro-BNP 3975, amylase / lipase 99/113. Gasometrical values on entrance: $\mathrm{pH}$ 7.05; pCO2 $15 \mathrm{mmHg}$; pO2 $95 \mathrm{mmHg}$; $\mathrm{Na}+129 \mathrm{mmol}$ / 1; $\mathrm{K}+5.9 \mathrm{mmol} / \mathrm{1}$ ). At this point admission on an Intermediate Care Unit was decided for evolutionary surveillance, etiological investigation and therapeutic orientation.

The clinical condition worsened on the first 24 hours of hospitalization, besides medical treatment of the AF with rapid ventricular response. Because of the symptoms consistent with acute heart failure, with hypotension $(72 / 43 \mathrm{mmHg})$ and marked peripheral hypoperfusion (capillary filling time> $4 \mathrm{sec}$ ), an emergent electrical cardioversion was performed for pace control. Concomitantly a "de novo" state of lethargy and confusion were observed, interpreted in the context of cerebral hypoperfusion and shock.

On the third day, a severe renal function with anuria and analytically a huge increase in INR (20.3) and liver cytolysis enzymes: AST 2293 U / L, ALT 876 $\mathrm{U} / \mathrm{L}$ and LDH $5731 \mathrm{U} / \mathrm{L}$ were detected.

At this point a diagnostic of acute liver failure of an etiology to be clarified was made. A large analytical study was conducted to exclude all the main causes of acute liver failure, specifically bacterial or viral infections, (cultures of blood, urine and serology's were all negative hepatotropic virus and other infectious agents), or underlying autoimmune pathology. The review of the clinical history and analytics allowed us to rule out the hypothesis of drug iatrogenesis or drug hypersensitivity and consumption or exposure to toxic products.

Imaging ultrasound and abdominal Doppler ultrasound did not show relevant morphological abnormalities.

After 7 days on the Intermediate Care Unit (ICU), and after hemodynamic stabilization with pace control of the $\mathrm{AF}$ and posterior compensation of the acute heart failure and shock, clinical and analytical parameters rapidly improved with resolution of the acute liver failure.

Based on the clinical and analytical evolution and the exclusion of other causes of acute hepatic necrosis, acute liver failure caused by ischemic hepatitis was assumed with the $\mathrm{AF}$ with rapid ventricular response as the most probable cause of the hemodynamic instability. The AF with a rapid ventricular response led to the hemodynamic changes that culminated in tissue hypoperfusion and hepatocyte ischemia.

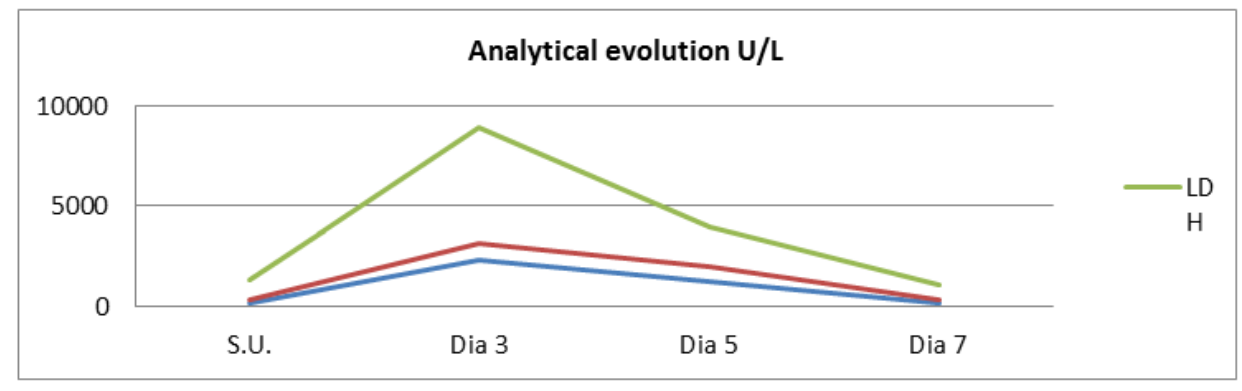

Analytical evolution of hepatic enzymes

\section{DiscuSsiON}

Ischemic hepatitis (IH) was initially described liver lesion with histological pattern of central necrosis distributed peripherally to central veins. in 1901 by F.B. Mallory (Boston City Hospital), as a 
Although IH is uncommon $\left(1 / 1000^{2}\right)$, it is known that its prevalence is amplified by 10 in critically ill patients, being a cause up to $2.5 \%$ [3] of admissions to the intensive care services.

The diagnosis is made in the presence of three fundamental conditions: i) heart or respiratory failure; (ii) sudden increase in liver cytolysis enzyme values which may reach and exceed 20 times the upper limit of normality; iii) exclusion of other causes of acute hepatic necrosis, namely drug-induced or infectious[5].

The histological pattern, now known as centrilobular hepatic necrosis and its associated clinical syndrome, known as ischemic hepatitis or hypoxic hepatitis [1], is analytically characterized by a sudden increase in plasma aminotransferases caused by anoxia and is usually associated with the underlying disease which in an acute manner, compromises hepatic hemodynamic stability and, consequently, decreases the availability of oxygen in hepatocytes [1].

Usually, patients with ischemic hepatitis are characterized by a low functional reserve, in more than $90 \%$ of the cases; the syndrome evolves in the context of respiratory failure, septic shock or heart failure, representing the latter between 39 and $70 \%$ of cases [1]. Most of the times it starts with a deterioration of patient's basal state by an acute event (e.g., arrhythmia, acute myocardial infarction or pulmonary thromboembolism) that triggers a global heart failure. Liver damage is suspected on the first day of admission, with elevated AST and LDH levels progressively increasing, with biochemical changes peaking between 48 and 72 hours... At early stages [4, 5]. some degree of behavioral changes (agitation or confusion) are possible, more likely resulting from cerebral hypoxia than acute liver failure

As previously mentioned in the first $72 \mathrm{~h}$ the analytical changes, are extraordinary. Liver enzyme increases, reaching values that can exceed 20 times the upper limit of normality, with particular attention to $\mathrm{LDH}$, whose elevation makes the differential diagnosis with viral hepatitis, more moderate. Some studies suggest that serum creatinine may also be an important marker in ischemic hepatitis, with increases exceeding 2 $\mathrm{mg} / \mathrm{dl}$ in $65 \%$ of cases or $5 \mathrm{mg} / \mathrm{dl}$ in $15 \%$ of patients included in the studies [4]. Renal dysfunction resulting from hemodynamic instability may be important for differential diagnosis as these changes are not common in viral or drug-induced hepatitis.

The biochemical pattern, being suggestive of ischemic hepatitis, is not pathognomonic and may also appear, in cases of acute centrilobular necrosis due to acetaminophen poisoning [7]. In this situation, enzymatic values and a clinical picture resolution occurs later, in about 10 to 15 days.
The diagnosis of ischemic hepatitis (IH) is usually simple when accompanied by a pattern of biochemical evolution that fits the correct clinical context. Obstacles to the correct and effective diagnosis are the absence of shock (which only happens in $50 \%$ of cases), unknown drug overdose and unknown cardiomyopathy. When ischemic hepatitis typically progresses, histological or imaging confirmation for diagnosis is not imperative [6], being the diagnosis possible with clinical and analytical data.

From a therapeutic point of view, hemodynamic stability is the main target for the improvement of the patient's clinical condition. The preservation of the hepatosplenic circulation is of prime importance. In cases of shock or congestive heart failure, blood flow is diverted to key organs such as the heart and brain, thereby aggravating the major lesion resulting from hepatocyte ischemia.

The prognosis of patients with ischemic hepatitis is poor with a mortality rate of $50 \%$ during the first days of intensive care unit stay $[1,2]$.

A severe and feared complication of ischemic hepatitis is acute liver failure, consisting of the development of acute liver injury, resulting in encephalopathy and impairment of liver synthesis function, which results in an increase in INR to 1.5 or greater, in a patient without pre-existing liver disease. Similar to hepatic ischemia, acute liver failure has a high morbidity and mortality rate (up to $80 \%$ ).

All patients with clinical or laboratory evidence of acute hepatitis should be promptly and carefully assessed for possible subtle changes in consciousness, and changes in coagulation tests should be sought analytically, including an increase in INR. If these criteria are met, the patient should be admitted to hospital, preferably in the intensive care unit (ICU), as this condition is expected to deteriorate rapidly. Early transfer to institutions with liver transplantation units may also be considered in selected cases.

Determining its etiology provides one of the best predictors of prognosis and allows guiding the therapy. A thorough clinical history is fundamental to ascertain the consumption of drugs or other potentially hepatotoxic products assisted by an extensive initial laboratory study. Special attention should be given to the study of liver coagulation and enzymes, which allows an assessment of the patient's clinical progression.

In patients with evidence of ischemic injury, cardiovascular support is the treatment of choice. The therapeutic success of heart failure or another cause of ischemia determines the end point result and in these cases, transplantation is rarely indicated. 
If the etiological diagnosis remains undefined after extensive initial evaluation, liver biopsy may be appropriate to try to identify a specific etiology that may influence the therapeutic strategy.

\section{Conclusion}

Ischemic hepatitis is nowadays a diagnostic challenge for internists/intensivists, particularly when the patient's signs and symptoms do not meet the most frequent clinical standard. The prognosis is unfavorable, with mortality reaching up to $50 \%$ of cases, occurring mainly in the first days after hospitalization, in most cases due to complications related to the severity of the underlying disease and not to isolated liver failure. There is not yet a specific treatment directed to liver pathology, being crucial the early diagnosis and stabilization of the underlying disease. Since acute liver failure is one of the most feared complications of hepatocyte ischemia, it should be early identified for better patient guidance on admission. The monitoring of severity and evolution of concomitant hepatic encephalopathy is crucial as it may motivate advanced life support measures. Depending on the degree of encephalopathy associated with the condition, the cerebral edema that often affects these patients also varies. In the most severe cases, grade IV hepatic encephalopathy, cerebral edema reaches from 65 to $75 \%$ of patients. In this context, ammonia is thought to play an important pathological role, with the administration of lactulose as a therapeutic option, both in the prevention and treatment of cerebral edema due to hepatic encephalopathy. The placement of an intracranial pressure probe (ICP) is also recommended to better control the evolution of the neurological condition and to prevent severe complications due to very high ICPs. The administration of mannitol for edema reduction and hyperventilation for $\mathrm{pCO} 2$ targets between 25 and $35 \mathrm{mmHg}$ are other therapeutic strategies to be adopted in the critically ill patient. In very severe and selected cases a surgical option is orthotopic liver transplantation.
The etiology and development of complications are the most important prognostic factors in acute liver failure. Patients with hepatic failure due to acetaminophen poisoning have a better prognosis compared to those without defined etiology and associated grade III/IV encephalopathy. The risk of mortality rises dramatically in the presence of complications such as cerebral edema, acute kidney injury, respiratory distress syndrome, coagulopathy or infection. The several therapeutical options available to treat these patients by intensive care medicine has led to a reduction in the mortality rate referred as $40-45 \%$ in most developed countries [10].

\section{Learning points}

- Ischemic hepatitis is a not so common diagnosis in the context of acute cardiac insufficiency.

- The authors provide an intensive literature review about this condition, its etiology, differential diagnosis, diagnostic options and treatments.

- This article highlights the importance of being aware of this diagnose, when approaching a decompensated heart failure patient, as emergency therapeutic measures clearly modify the prognosis.

\section{REFERENCES}

1. J H. Hypoxic hepatitis. Liver international. 2011; 2(12).

2. Chang JP TCK. Serum albumin and male gender are independent predictors of mortality in patients with hypoxic hepatitis and can be used in a prognostic model to predict early in-patient mortality. Hepatology. 2008; 48(Suppl 447-A).

3. JM R. Hypoxic hepatitis in critically ill patients: incidence, etiology and risk factors for mortality. J. Anesth. 2011; 25(50-6).

4. Henrion J SM. Hypoxic hepatitis. Clinical and hemodynamic study in 142 consecutive cases. Medicine. 2003; 82(392-406).

5. Fuhrmann $\mathrm{V}$ ea. Hypoxic hepatitis: underlying conditions and risk factors dor mortality in critically ill patients. Intensive Care Med. 2009; 35(1397-405). 